\title{
The Prognostic Relevance of Psammoma Bodies and Ultrasonographic Intratumoral Calcifications in Papillary Thyroid Carcinoma: Reply
}

\author{
Jung-Soo Pyo $\cdot$ Guhyun Kang $\cdot$ Dong-Hoon Kim • \\ Chanheun Park · Joo Heon Kim • Jin Hee Sohn
}

Published online: 1 October 2013

(C) Société Internationale de Chirurgie 2013

\begin{abstract}
All 258 patients were diagnosed as classical variant of papillary thyroid carcinoma (PTC) in the present study. In 2010, at our institution (The Breast and Thyroid Cancer Center of Kangbuk Samsung Hospital), follicular variant of PTC was diagnosed in six patients; however, no patient was diagnosed as other variants, including tall cell and columnar cell variants. Thus, we did not include other variants due to too small cases. We excluded patients without ultrasonographic findings on our institution; however, no other exclusion criterion was present. In the present study, other variants of PTC, except classical variant, were not included, so we do not have any data about the correlation between aggressive variants and psammoma bodies. We did not investigate for fineneedle aspiration cytology (FNAC) finding in the present study; therefore, we do not have any data or information for
\end{abstract}

Jung-Soo Pyo and Guhyun Kang have contributed equally to both the reply to letter to the editor and the original article.

J.-S. Pyo · D.-H. Kim · J. H. Sohn ( $\varangle)$

Department of Pathology, Kangbuk Samsung Hospital,

Sungkyunkwan University School of Medicine, 78 Saemunan-

gil, Jongno-gu, Seoul 110-746, South Korea

e-mail: jhpath.sohn@samsung.com

G. Kang

Department of Pathology, Sanggye Paik Hospital, Inje

University College of Medicine, Seoul, South Korea

C. Park

Department of Surgery, Kangbuk Samsung Hospital,

Sungkyunkwan University School of Medicine, Seoul,

South Korea

J. H. Kim

Department of Pathology, Eulji University Hospital, Eulji

University School of Medicine, Taejon, South Korea incidence of psammoma bodies in FNAC. Three specialists in the radiology department have performed ultrasonographic evaluation and did ultrasonography-guided aspiration by the same radiologist in case of suspicious malignancy in our institution; ultrasonography for 258 patients also was evaluated by them. The important ultrasonographic diagnostic criteria of suspicious malignancy used in our institution included tumor margin, shape, echogenicity, and intratumoral calcification. However, whether intratumoral calcification targeted or not could not be confirmed at present. Therapeutic or prophylactic central-compartment neck dissection was performed according to Recommendation 27(a) to (c) in the American Thyroid Association guidelines [1]. The surgeons clearly dissected the ipsilateral central neck dissection on hemithyroidectomy.

\section{References}

1. Cooper DS, Doherty GM, Haugen BR et al (2009) Revised American Thyroid Association management guidelines for patients with thyroid nodules and differentiated thyroid cancer. Thyroid 19:1167-1214 